\title{
STRONGLY DISSIPATIVE OPERATORS AND NONLINEAR EQUATIONS. IN A FRÉCHET SPACE
}

\author{
R. H. MARTIN, JR.
}

\begin{abstract}
Suppose that $X$ is a Fréchet space, $Y$ is a Banach subspace of $X$, and $A$ is a function from $Y$ into $X$. Sufficient conditions are determined to insure that the equation $A x=y(y \in Y)$ has a unique solution $x_{y}$ which depends continuously on $y$. The techniques of this paper use the theory of dissipative operators in a Banach space, and the results are associated with the idea of admissibility of the space $Y$. Also, the equation $A x=C x+y$ is considered where $C$ is completely continuous.
\end{abstract}

Let $X$ be a Fréchet space over the real or complex field (i.e., $X$ is a locally convex, complete, metrizable topological vector space-see e.g., $[10$, p. 85]). In this paper we assume the following:

(X1) $\left(q_{n}\right)_{1}^{\infty}$ is an increasing family of continuous seminorms on $X$ which defines the topology of $X$ (i.e., $q_{n} \leqq q_{n+1}$ and $X-\lim _{k \rightarrow \infty} x_{k}=x$ if and only if $\lim _{k \rightarrow \infty} q_{n}\left(x_{k}-x\right)=0$ for each $\left.n\right)$.

(X2) $Y=\left\{x \in X: \sup _{n}\left\{q_{n}(x)\right\}<\infty\right\}$ and $|x|=\sup _{n}\left\{q_{n}(x)\right\}$ for each $x$ in $Y$.

Note that a sequence of seminorms satisfying (X1) always exists, and the space $Y$ in $(\mathrm{X} 2)$ is a complete normed space with norm $|\cdot|$. However, the members of $Y$ depend on how the sequence $\left(q_{n}\right)_{1}^{\infty}$ is chosen.

In this paper, some results on strongly dissipative operators in a Banach space are used to establish analogous results for a class of operators which map $Y$ into $X$. Recently, some fixed point theorems for completely continuous perturbations of Lipschitz continuous functions in locally convex spaces have been obtained by Cain and Nashed [1]. In this paper, a class of functions $A$ from $Y$ into $X$ is considered which can be "approximated" by functions which are defined on $X$. Sufficient conditions are established to insure that the equation $A x=y$ has a unique solution $x_{y}$ for each $y$ in $Y$, and the function $B$ defined by $B y=x_{y}$ for each $y$ in $Y$ has certain continuity properties. This result is closely associated with the notion of admissibility introduced by Massera and Schäffer [6]. Recently, admissibility has been used in studying existence and stability of solutions to Volterra integral equations-see Corduneanu [2], [3] and Miller [8].

Received by the editors May 3, 1971.

AMS 1969 subject classifications. Primary 4780; Secondary 4785.

Key words and phrases. Fréchet space, dissipative operator, nonlinear operator equation, admissibility.

(c) American Mathematical Society 1972 
We also consider the solvability of the equation $A x=C x+y$ where $C$ is a function from $Y$ into $Y$ which has certain compact properties and growth properties. Finally, two examples of integral equations on the half-line are given to illustrate when these techniques may be applied.

Definition 1. If $A$ is a function from $X$ into $X$, then $A$ is said to be compatible with $q_{n}$ if whenever $x$ is in $X$ and $\left(x_{k}\right)_{1}^{\infty}$ is a sequence in $X$ such that $\lim _{k \rightarrow \infty} q_{n}\left(x_{k}-x\right)=0$, it follows that $\lim _{k \rightarrow \infty} q_{n}\left(A x_{k}-A x\right)=0$.

For each positive integer $n$ let $M_{n}=\left\{x \in X: q_{n}(x)=0\right\}$ and let $X \mid M_{n}=$ $\left\{\phi(x): x \in X\right.$ and $\left.\phi(x)=x+M_{n}\right\}$. If $q_{n}^{*}(\phi(x))=q_{n}(x)$ for each $\phi(x)$ in $X / M_{n}$, then $q_{n}^{*}$ is well defined and is a norm on the quotient space $X / M_{n}$. If $A$ is a function from $X$ into $X$ which is compatible with $q_{n}$, then define $A^{*} \phi(x)=\phi(A x)$ for each $\phi(x)$ in $X / M_{n}$. It is easy to see that $A^{*}$ is well defined and continuous on $X \mid M_{n}$.

Definition 2. Suppose that $X / M_{n}, A$ and $A^{*}$ are as in the above paragraph and let $E_{n}$ denote the completion of the normed space $X / M_{n}$ with the norm on $E_{n}$ denoted by $q_{n}^{\prime}$. Then $A$ is said to be strongly dissipative with respect to $q_{n}$ if there is a continuous function $\alpha_{n}$ from $[0, \infty)$ into $[0, \infty)$ and a continuous function $A^{\prime}$ from $E_{n}$ into $E_{n}$ such that $\alpha_{n}(0)=0, \alpha_{n}(s)>0$ if $s>0, A^{*} \phi(x)=A^{\prime} \phi(x)$ if $\phi(x)$ is in $X / M_{n}$, and

$$
\lim _{h \rightarrow 0+}\left[q_{n}^{\prime}\left(x-y+h\left(A^{\prime} x-A^{\prime} y\right)\right)-q_{n}^{\prime}(x-y)\right] / h \leqq-\alpha_{n}\left(q_{n}^{\prime}(x-y)\right)
$$

for all $x$ and $y$ in $E_{n}$.

REMARK. In many cases the quotient spaces $X / M_{n}$ are completefor example, the spaces considered in Examples 1 and 2 below have this property. If $X / M_{n}$ is complete and $A$ is compatible with $q_{n}$, note that $A$ is strongly dissipative with respect to $q_{n}$ only in case

$$
\lim _{h \rightarrow 0+}\left[q_{n}(x-y+h(A x-A y))-q_{n}(x-y)\right] / h \leqq-\alpha_{n}\left(q_{n}(x-y)\right)
$$

for all $x$ and $y$ in $X$. Also, if the function $A^{*}$ is uniformly continuous on bounded subsets of $X / M_{n}$, then $A^{*}$ can be extended to a continuous function $A^{\prime}$ on $E_{n}$, and it can be shown that $A$ is strongly dissipative with respect to $q_{n}$.

Lemma 1. Suppose that $A$ is a function from $X$ into $X$ which is strongly dissipative with respect to $q_{n}$. Then, for each $z$ in $X$ and each $\varepsilon>0$, there is a $x_{z}^{\varepsilon}$ in $X$ such that $q_{n}\left(A x_{z}^{\varepsilon}-z\right) \leqq \varepsilon$.

Indication of Proof. If the function $A^{\prime}$ is as in Definition 2, it is easy to see that $A^{\prime}$ satisfies each of the suppositions of Theorem 2 in [7], and so there is a unique $y^{\prime}$ in $E_{n}$ such that $q_{n}^{\prime}\left(A^{\prime} y^{\prime}-\phi(z)\right)=0$. Since $X / M_{n}$ is dense in $E_{n}$ and $A^{\prime}$ is continuous, there is an $x_{z}^{\varepsilon}$ in $X$ such that 
$q_{n}^{\prime}\left(A^{\prime} \phi\left(x_{z}^{\varepsilon}\right)-\phi(z)\right) \leqq \varepsilon$. Thus, $q_{n}\left(A x_{x}^{\varepsilon}-z\right)=q_{n}^{*}\left(A^{*} \phi\left(x_{z}^{\varepsilon}\right)-\phi(z)\right) \leqq \varepsilon$ and the lemma is true.

We now prove our main result.

THeOrem 1. Suppose that conditions (X1)-(X2) are fulfilled, $\alpha$ is a continuous, increasing function from $[0, \infty)$ into $[0, \infty)$ such that $\alpha(0)=0$ and $\lim _{s \rightarrow \infty} \alpha(s)=\infty, A$ is a function from $Y$ into $X$, and $\left(A_{n}\right)_{1}^{\infty}$ is a sequence of functions from $X$ into $X$ such that

(i) $A_{n}$ is strongly dissipative with respect to $q_{n}$ for each $n$;

(ii) $\lim _{h \rightarrow 0+}\left[q_{n}\left(x-y+h\left(A_{n} x-A_{n} y\right)\right)-q_{n}(x-y)\right] / h \leqq-\alpha\left(q_{n}(x-y)\right)$ for each $x$ and $y$ in $X$ and each $n$;

(iii) there is a number $L \geqq 0$ such that $q_{n}\left(A_{n} 0\right) \leqq L$ for each $n$;

(iv) for each pair of positive numbers $K$ and $\delta$ there is a positive integer $N(K, \delta)$ such that if $j \geqq i \geqq N(K, \delta)$ and $x$ is a member of $X$ such that $q_{j}(x) \leqq K$, then $q_{i}\left(A_{i} x-A_{j} x\right) \leqq \delta ;$ and

(v) if $K$ is a positive number, then $\lim _{i \rightarrow \infty} q_{i}\left(A_{i} x-A x\right)=0$, uniformly for $x$ in $Y$ with $|x| \leqq K$.

Then, for each $z$ in $Y$, there is a unique $x_{z}$ in $Y$ such that $A x_{z}=z$. Furthermore, if the function $B$ from $Y$ into $Y$ is defined by $B z=x_{z}$ for each $z$ in $Y$, then $|B z-B w| \leqq \alpha^{-1}(|z-w|)$ for all $z$ and $w$ in $Y$.

Remark. Note that the function $A$ in Theorem 1 need not be defined on all of $X$ and does not necessarily map $Y$ into $Y$. Furthermore, by supposition (ii) if the quotient spaces $X / M_{n}$ are complete for each $n$, then we need only assume that $A_{n}$ is compatible with $q_{n}$ in supposition (i)-see remark following Definition 2.

Proof of Theorem 1. Note that supposition (ii) implies that

$$
q_{n}(x-y) \leqq \alpha^{-1}\left(q_{n}\left(A_{n} x-A_{n} y\right)\right)
$$

for all $x$ and $y$ in $X$. By Lemma 1 and suppositions (i) and (ii), there is an $x_{z}^{n}$ in $X$ such that $q_{n}\left(A_{n} x_{z}^{n}-z\right) \leqq n^{-1}$ for each $z$ in $Y$ and positive integer $n$. Since $\alpha^{-1}$ is increasing, we have from (1) and supposition (iii) that

$$
q_{n}\left(x_{z}^{n}\right) \leqq \alpha^{-1}\left(q_{n}\left(A_{n} x_{z}^{n}-A_{n} 0\right)\right) \leqq \alpha^{-1}(|z|+1+L)
$$

for each $n$. Let $\varepsilon$ be a positive number and let $\delta>0$ be such that $\alpha^{-1}(s) \leqq \varepsilon$ whenever $0 \leqq s \leqq 2 \delta$. If $N$ is as in (iv) and $j \geqq i \geqq N\left(\alpha^{-1}(|z|+1+L), \delta\right)$, then $q_{j}\left(x_{z}^{j}\right) \leqq \alpha^{-1}(|z|+1+L)$, so

$$
\begin{aligned}
q_{i}\left(A_{i} x_{z}^{i}-A_{i} x_{z}^{j}\right) & \leqq q_{i}\left(z-A_{i} x_{z}^{j}\right)+i^{-1} \\
& \leqq q_{i}\left(z-A_{j} x_{z}^{j}\right)+q_{i}\left(A_{j} x_{z}^{j}-A_{i} x_{z}^{j}\right)+i^{-1} \\
& \leqq 2 i^{-1}+q_{i}\left(A_{j} x_{z}^{j}-A_{i} x_{z}^{j}\right) \leqq 2 i^{-1}+\delta
\end{aligned}
$$


Now let $m$ be a positive integer such that

$$
m \geqq \max \left\{N\left(\alpha^{-1}(|z|+1+L), \delta\right), 2 / \delta\right\} .
$$

If $j \geqq i \geqq m$, then by (1), (3), and the choice of $\delta$,

$$
q_{i}\left(x_{z}^{i}-x_{z}^{j}\right) \leqq \alpha^{-1}\left(q_{i}\left(A_{i} x_{z}^{i}-A_{i} x_{z}^{j}\right)\right) \leqq \alpha^{-1}(2 \delta) \leqq \varepsilon .
$$

It now follows easily from (4) and (X1) that $\left(x_{z}^{k}\right)_{1}^{\infty}$ is a Cauchy sequence in $X$. Since $X$ is complete, there is an $x_{z}$ in $X$ such that $X-\lim _{k \rightarrow \infty} x_{z}^{k}=x_{z}$. By (2),

$$
q_{n}\left(x_{z}\right)=\lim _{k \rightarrow \infty} q_{n}\left(x_{z}^{k}\right) \leqq \limsup _{k \rightarrow \infty} q_{k}\left(x_{z}^{k}\right) \leqq \alpha^{-1}(|z|+1+L)
$$

for each $n$, so $x_{z}$ is in $Y$ with $\left|x_{z}\right| \leqq \alpha^{-1}(|z|+1+L)$. Letting $j \rightarrow \infty$ in the term on the left side of (3) shows that $\lim _{i \rightarrow \infty} q_{i}\left(A_{i} x_{z}^{i}-A_{i} x_{z}\right)=0$. Thus, from supposition ( $v)$, if $n$ is a positive integer,

$$
\begin{aligned}
q_{n}\left(A x_{z}-z\right) & \leqq \lim _{i \rightarrow \infty} q_{i}\left(A x_{z}-z\right)=\lim _{i \rightarrow \infty} q_{i}\left(A_{i} x_{z}-z\right) \\
& \leqq \lim _{i \rightarrow \infty} q_{i}\left(A_{i} x_{z}-A_{i} x_{z}^{i}\right)+i^{-1}=0 .
\end{aligned}
$$

Hence $A x_{z}=z$. If $y$ is in $Y$ and $A y=z$, then by (1) and supposition (v),

$$
\begin{aligned}
q_{n}\left(y-x_{z}\right) & \leqq \lim _{i \rightarrow \infty} q_{i}\left(y-x_{z}\right) \leqq \lim _{i \rightarrow \infty} \alpha^{-1}\left(q_{i}\left(A_{i} y-A_{i} x_{z}\right)\right) \\
& \leqq \lim _{i \rightarrow \infty} \alpha^{-1}\left(q_{i}\left(A_{i} y-z\right)+q_{i}\left(z-A_{i} x_{z}\right)\right)=\alpha^{-1}(0)=0
\end{aligned}
$$

for each $n$. Consequently $y=x_{z}$ and the function $B$ defined in the statement of the theorem is well defined. Furthermore, if $z$ and $w$ are in $Y$ and $\left(x_{z}^{i}\right)_{1}^{\infty}$ and $\left(x_{w}^{i}\right)_{1}^{\infty}$ are as constructed above, then by (1) and the fact that $q_{i}\left(A_{i} x_{z}^{i}-A_{i} x_{w}^{i}\right) \leqq q_{i}(z-w)+2 i^{-1}$,

$$
\begin{aligned}
q_{n}\left(x_{z}-x_{w}\right) & =\lim _{i \rightarrow \infty} q_{n}\left(x_{z}^{i}-x_{w}^{i}\right) \leqq \limsup _{i \rightarrow \infty} q_{i}\left(x_{z}^{i}-x_{w}^{i}\right) \\
& \leqq \limsup _{i \rightarrow \infty} \alpha^{-1}\left(q_{i}\left(A_{i} x_{z}^{i}-A_{i} x_{w}^{i}\right)\right) \\
& \leqq \limsup _{i \rightarrow \infty} \alpha^{-1}\left(q_{i}(z-w)+2 i^{-1}\right) \leqq \alpha^{-1}(|z-w|) .
\end{aligned}
$$

Thus,

$$
|B z-B w|=\sup \left\{q_{n}\left(x_{z}-x_{v}\right): n=1,2, \cdots\right\} \leqq \alpha^{-1}(|z-w|)
$$

and the proof of Theorem 1 is complete.

LEMMA 2. Let the suppositions of Theorem 1 be fulfilled and for each $R>0$ let $Q_{R}=\{x \in Y:|x| \leqq R\}$. Then, considering $Q_{R}$ with the topology induced by $X$, the function $B$ defined in Theorem 1 is continuous from $Q_{R}$ into $X$. 
Indication of Proof. Let $x$ be in $Q_{R}$ and let $\left(x_{k}\right)_{1}^{\infty}$ be a sequence in $Q_{R}$ such that $\lim _{k \rightarrow \infty} q_{n}\left(x_{k}-x\right)=0$ for each $n$. Let $n$ be a positive integer, let $\varepsilon>0$, and let $\delta>0$ be such that $\alpha^{-1}(s) \leqq \varepsilon$ whenever $0 \leqq s \leqq \delta$. Note that if $y$ is in $Q_{R}$, then $|B y| \leqq|B y-B O|+|B O| \leqq \alpha^{-1}(R)+|B O|$. By supposition (v) of Theorem 1, let the integer $m \geqq n$ be such that $q_{m}\left(A_{m} y-A y\right) \leqq \delta / 3$ for all $y$ in $Y$ with $|y| \leqq \alpha^{-1}(R)+|B O|$. Let $p$ be a positive integer such that $q_{m}\left(x_{k}-x\right) \leqq \delta / 3$ whenever $k \geqq p$. If $k \geqq p$, we have from (1), the choice of $\delta$, and the fact that $\alpha^{-1}$ is increasing, that

$$
\begin{aligned}
q_{n}\left(B x_{k}-B x\right) & \left.\leqq q_{m}\left(B x_{k}-B x\right) \leqq \alpha^{-1}\left(q_{m}{ }^{\prime} A_{m} B x_{k}-A_{m} B x\right)\right) \\
& \leqq \alpha^{-1}\left(q_{m}\left(A_{m} B x_{k}-A B x_{k}\right)+q_{m}\left(A B x_{k}-A B x\right)\right. \\
& \left.\quad+q_{m}\left(A B x-A_{m} B x\right)\right) \\
& \leqq \alpha^{-1}\left(\delta / 3+q_{m}\left(x_{k}-x\right)+\delta / 3\right) \leqq \alpha^{-1}(\delta) \leqq \varepsilon .
\end{aligned}
$$

Thus $\lim _{k \rightarrow \infty} q_{n}\left(B x_{k}-B x\right)=0$ for each $n$, and the assertion of the lemma follows.

THEOREM 2. Let the suppositions of Theorem 1 be fulfilled and suppose that $C$ is a function from $Y$ into $Y$ such that

$$
\limsup _{|x| \rightarrow \infty} \alpha^{-1}(|C x|) /|x|=\beta<1 .
$$

Suppose further that at least one of the following is satisfied:

(i) $C$ is continuous from the Banach space $Y$ into itself and maps bounded subsets of $Y$ into relatively compact subsets of $Y$; or

(ii) if $Q_{R}$ is as in Lemma 2 (with the topology induced by $X$ ), then, for each $R>0, C$ is continuous from $Q_{R}$ into $X$ and the image of $Q_{R}$ under $C$ is relatively compact in $X$.

Then, for each $z$ in $Y$, there is a $y_{z}$ in $Y$ such that $A y_{z}-C y_{z}=z$.

Indication of Proof. Note that we need only show that there is an $x_{0}$ in $Y$ such that $A x_{0}-C x_{0}=0$, or equivalently, $B \cdot C x_{0}=x_{0}$ where $B$ is as defined in Theorem 1. Since $\lim \sup _{|x| \rightarrow \infty}|B \cdot C x| /|x|<1$, let $r_{1}>0$ be such that $|B \cdot C x| \leqq|x|$ whenever $|x| \geqq r_{1}$ and let $r_{2}=\sup \left\{|B \cdot C x|:|x| \leqq r_{1}\right\}$. If $R=\max \left\{r_{1}, r_{2}\right\}$, then $B \cdot C$ maps $Q_{R}$ into $Q_{R}$. If (i) holds the theorem follows from the Schauder fixed point theorem, and if (ii) holds the theorem follows from Lemma 2 and the Tychonov fixed point theorem (see e.g. [5, Theorem 5, p. 456]).

EXAMPLE 1. Let $X$ be the space $L L^{2}[0, \infty)$ of all measurable functions $x$ from $[0, \infty)$ into the real numbers such that $q_{n}(x)=\left(\int_{0}^{n}|x(s)|^{2} d s\right)^{1 / 2}<\infty$ for each positive integer $n$. Note that $Y$ is the space $L^{2}[0, \infty)$ and $|x|=\left(\int_{0}^{\infty}|x(s)|^{2} d s\right)^{1 / 2}$ for each $x$ in $Y$. Let $a$ and $b$ be symmetric, measurable, locally integrable functions defined on $[0, \infty)^{2}$ such that the operator 
$T x(t)=\int_{0}^{t} a(t, s) x(s) d s$ maps $L L^{2}[0, \infty)$ continuously into $L L^{2}[0, \infty)$ and the operator $S x(t)=\int_{0}^{\infty} b(t, s) x(s)$ maps $L^{2}[0, \infty)$ into $L^{2}(0, \infty)$. Assume further that (a) $\int_{0}^{n} \int_{0}^{n} a(t, s) x(s) x(t) d s d t \geqq 0$ for each $x$ in $L L^{2}[0, \infty)$ and each $n$; (b) $\int_{0}^{n} \int_{0}^{n} b(t, s) x(s) x(t) d s d t \geqq 0$ for each $x$ in $L L^{2}[0, \infty)$ and each $n$; and (c) $\int_{0}^{\infty} \int_{0}^{\infty}|b(t, s)|^{2} d s d t<\infty$. Define $A x=-x-T x-S x$ for each $x$ in $L^{2}[0, \infty)$. Then the suppositions of Theorem 1 are fulfilled with $\alpha(s)=-s$ for all $s \geqq 0$ and $A_{n} x=-x-T x-S_{n} x$ where $S_{n} x(t)=\int_{0}^{n} b(t, s) x(s) d s$ for each $x$ in $L L^{2}[0, \infty)$. Suppositions (i) and (iii) of Theorem 1 are easily seen to be true. Supposition (ii) is immediate from (a) and (b) above and the fact that

$$
2 \int_{0}^{n}\left[\int_{0}^{t} a(t, s) x(s) d s\right] x(t) d t=\int_{0}^{n} \int_{0}^{n} a(t, s) x(s) x(t) d s d t
$$

which follows from the symmetry of $a$. Since condition (c) above implies $\lim _{p \rightarrow \infty} \int_{0}^{p} \int_{p}^{\infty}|b(t, s)|^{2} d s d t=0$, it is easy to see that suppositions (iv) and (v) are fulfilled. Thus, for each $z$ in $L^{2}[0, \infty)$, there is a unique $x_{z}$ in $L^{2}[0, \infty)$ such that

$$
x_{z}(t)+\int_{0}^{t} a(t, s) x_{z}(s) d s+\int_{0}^{\infty} b(t, s) x_{z}(s) d s=z(t)
$$

for almost all $t$ in $[0, \infty)$. Also, $\int_{0}^{\infty}\left|x_{z}(s)\right|^{2} d s \leqq \int_{0}^{\infty}|z(s)|^{2} d s$. Note the operator defined by the left side of $(5)$ is not necessarily defined on all of $L L^{2}(0, \infty)$ and need not map $L^{2}[0, \infty)$ into itself.

Example 2. Let $C_{c}[0, \infty)$ denote the Fréchet space of all continuous functions $x$ from $\left[0, \infty\right.$ ) into the $m$ dimensional space $R^{m}$ (with $\|\cdot\|$ denoting an appropriate norm on $R^{m}$ ) with the topology generated by uniform convergence on compact subsets of $[0, \infty)$. Define $q_{n}(x)=$ $\max \{\|x(t)\|: 0 \leqq t \leqq n\}$ for each $x$ in $C_{c}[0, \infty)$ and each positive integer $n$. Then $Y$ is the space $B C[0, \infty)$ of all $x$ in $C_{c}[0, \infty)$ such that $|x|=$ $\sup \{\|x(t)\|: t \geqq 0\}<\infty$. Now let $f$ and $g$ be continuous functions from $[0, \infty)^{2} \times R^{m}$ into $R^{m}$ such that (a) $f(t, s, 0)=g(t, s, 0)=0$ for all $(t, s)$ in $[0, \infty)^{2}$; (b) $f(t, s, \xi)=0$ for all $(t, s, \xi)$ in $[0, \infty)^{2} \times R^{m}$ with $s>t$; and there are continuous functions $\theta$ and $\phi$ from $[0, \infty)^{2}$ into $[0, \infty)$ such that (c) $\sup \left\{\int_{0}^{t} \theta(t, s) d s: t \geqq 0\right\}=\lambda<1$; (d) $\sup \left\{\int_{0}^{\infty} \phi(t, s) d s: t \geqq 0\right\}=\gamma<1-\lambda$; (e) $\left\|f\left(t, s, \xi_{1}\right)-f\left(t, s, \xi_{2}\right)\right\| \leqq \theta(t, s)\left\|\xi_{1}-\xi_{2}\right\|$ for all $\left(t, s, \xi_{1}\right)$ and $\left(t, s, \xi_{2}\right)$ in $[0, \infty)^{2} \times R^{m}$; and (f) $\|g(t, s, \xi)\| \leqq \phi(t, s)\|\xi\|$ for all $(t, s, \xi)$ in $[0, \infty)^{2} \times R^{m}$. If $S$ is the integral operator defined on $C_{c}[0, \infty)$ by $S x(t)=$ $\int_{0}^{t} f(t, s, x(s)) d s$, then the suppositions of Theorem 1 are easily seen to be fulfilled with $A=-x+S x$ for each $x$ in $B C[0, \infty), A_{n} x=-x+S x$ for each $x$ in $C_{c}[0, \infty)$, and $\alpha(s)=(1-\lambda) s$ for each $s \geqq 0$. Let $T$ be the integral operator defined in $B C[0, \infty)$ by $T x(t)=\int_{0}^{\infty} g(t, s, x(s)) d s$ and suppose that $T$ maps $B C[0, \infty)$ into $B C[0, \infty)$. Note that conditions (d) and (f) 
above imply that $|T x| \leqq \gamma|x|$ for all $x$ in $B C[0, \infty)$. Now let $z$ be in $B C[0, \infty)$ and let $C x=T x+z$ for each $x$ in $B C[0, \infty)$. If $B$ is as in Theorem 1 (i.e., $A \cdot B x=x$ for all $x$ in $B C[0, \infty)$ ), then $B O=0$ and we have that $|B x| \leqq(1-\lambda)^{-1}|x|$ for all $x$ in $B C[0, \infty)$. It now follows easily that the operator $B \cdot C$ maps the ball $Q=\left\{x \in B C[0, \infty):|x| \leqq|z|(1-\gamma-\lambda)^{-1}\right\}$ into itself. Thus, if $C$ is completely continuous for the $B C[0, \infty)$ topology on $Q$ or if $C$ is completely continuous for the $C_{c}[0, \infty)$ topology on $Q$ (note that this is the case if

$$
\lim _{p \rightarrow \infty}\left[\sup \left\{\int_{p}^{\infty} \phi(t, s) d s: 0 \leqq t \leqq p\right\}\right]=0,
$$

where $\phi$ is as in (d)), then there is an $x_{z}$ in $B C[0, \infty)$ with

such that

$$
\left|x_{z}\right| \leqq|z|(1-\gamma-\lambda)^{-1}
$$

$$
x_{z}(t)-\int_{0}^{t} f\left(t, s, x_{z}(s)\right) d s=\int_{0}^{\infty} g\left(t, s, x_{z}(s)\right) d s+z(t)
$$

for all $t$ in $[0, \infty)$. Furthermore, since $|B \cdot C x|<|x|$ if $|x|>|z|(1-\gamma-\lambda)^{-1}$, all solutions $x_{z}$ to (6) satisfy $\left|x_{z}\right| \leqq|z|(1-\gamma-\lambda)^{-1}$. In particular $\left|x_{z}\right| \rightarrow 0$ as $|z| \rightarrow 0$, so we have a type of stability criteria for the zero solution of equation (6) when $z(t)=0$ for all $t \geqq 0$.

REMARK. In Example 2, we need only assume that the inequalities (e) and (f) hold in some neighborhood of the origin in $R^{m}$. This follows from the fact that if $r>0$ and $h$ is a function from $Q_{r}=\left\{\xi \in R^{m}:\|\xi\| \leqq r\right\}$ into $R^{m}$ such that $\left\|h\left(\xi_{1}\right)-h\left(\xi_{2}\right)\right\| \leqq k\left\|\xi_{1}-\xi_{2}\right\|$ for all $\xi_{1}$ and $\xi_{2}$ in $Q_{r}$, then there is a function $h^{*}$ from $R^{m}$ into $R^{m}$ such that $h^{*}(\xi)=h(\xi)$ for all $\xi$ in $Q_{r}$ and $\left\|h^{*}\left(\xi_{1}\right)-h^{*}\left(\xi_{2}\right)\right\| \leqq k^{*}\left\|\xi_{1}-\xi_{2}\right\|$ for all $\xi_{1}$ and $\xi_{2}$ in $R^{m}$ where $k \leqq k^{*} \leqq 2 k$ (take $h^{*}(\xi)=h(r \xi /\|\xi\|)$ if $\left.\|\xi\|>r\right)$. Note that $k^{*}=k$ if $\|\cdot\|$ is the Euclidean norm on $R^{m}$. Thus the stability and existence criteria established in Example 2 give some improvements to those of Miller, Nohel and Wong [9].

\section{REFERENCES}

1. G. L. Cain, Jr. and M. Z. Nashed, Fixed points and stability for a sum of two operators in locally convex spaces, Pacific J. Math. (to appear).

2. C. Corduneanu, Problèmes globaux dans la théorie des équations intégrales de Volterra, Ann. Mat. Pura Appl. (4) 67 (1965), 349-363. MR 32 \#331.

3. - Some perturbation problems in the theory of integral equations, Math. Systems Theory 1 (1967), 143-155. MR 35 \#4773.

4. J. Dieudonné, Foundations of modern analysis, Pure and Appl. Math., Vol. 10, Academic Press, New York, 1960. MR 22 \#11074.

5. N. Dunford and J. T. Schwartz, Linear operators. I: General theory, Pure and Appl. Math., vol. 7, Interscience, New York and London, 1958. MR 22 \#8302.

6. J. L. Massera and J. J. Schäffer, Linear differential equations and function spaces, Pure and Appl. Math., vol. 21, Academic Press, New York, 1966. MR 35 \#3197. 
7. R. H. Martin, Jr., Lyapunov functions and autonomous differential equations in a Banach space, Math. Systems Theory (to appear).

8. R. K. Miller, Admissibility and nonlinear Volterra integral equations, Proc. Amer. Math. Soc. 25 (1970), 65-71. MR 41 \#2324.

9. R. K. Miller, J. A. Nohel and J. S. W. Wong, A stability theorem for nonlinear mixed integral equations, J. Math. Anal. Appl. 25 (1969), 446-449. MR 38 \#2566.

10. F. Trèves, Topological vector spaces, distributions, and kernels, Academic Press, New York, 1967. MR 37 \#726.

Department of Mathematics, North Carolina State University, Raleigh, North Carolina 27607 